\title{
Effectiveness and Response Predictors of Omalizumab in Treating Patients with Seasonal Allergic Rhinitis: A Real-World Study
}

This article was published in the following Dove Press journal:

Journal of Asthma and Allergy

\author{
Tingting $\mathrm{Ma}^{\mathrm{I}}$ \\ Hongtian Wang ${ }^{2}$ \\ Xueyan Wang'
}

'Department of Allergy, Beijing Shijitan Hospital, Capital Medical University, Beijing Key Laboratory of BioCharacteristic Profiling for Evaluation of Rational Drug Use, Beijing 100038, People's Republic of China; ${ }^{2}$ Department of Otolaryngology Head and Neck Surgery, Beijing Shijitan Hospital, Capital Medical University, Beijing 100038,

People's Republic of China
Correspondence: Hongtian Wang Department of Otolaryngology Head and Neck Surgery, Beijing Shijitan Hospital,

Capital Medical University, No. 10, Tieyi Road, Haidian District, Beijing I00038,

People's Republic of China

Tel +86 13391836668

Email wht30I@263.net

Xueyan Wang

Department of Allergy, Beijing Shijitan Hospital, Capital Medical University,

Beijing Key Laboratory of Biocharacteristic Profiling for Evaluation of Rational Drug Use, No. 10, Tieyi Road, Haidian District, Beijing 100038, People's Republic of China

Tel +86 |39| |6I2465

Email wangxueyan2018@163.com
Background: Omalizumab has been proven effective and safety in treating seasonal allergic rhinitis (SAR) by several randomized clinical trials in many countries. However, there is lack of clinical reports of Chinese patients with SAR treated by omalizumab.

Objective: In the present real-world-designed study, we aimed to investigate the effectiveness of omalizumab in treating patients with SAR.

Methods: SAR patients administered omalizumab in various dosages were recruited, and follow-up was done. Their quality of life (QOL) and symptoms were assessed by the Rhinoconjunctivitis Quality of Life Questionnaire (RQLQ), Total Nasal Symptoms Score (TNSS), Asthma Control Test (ACT), and clinical outcomes were compared between postand pre-treatment conditions.

Results: Sixty SAR patients received omalizumab therapy in the study (mean age 35.47 \pm 17.02 years, 35 females). Omalizumab treatment significantly improved the quality of life (change in RQLQ overall score: $-2.08 \pm 1.01$, paired $t$-test $\mathrm{p}<0.001$ ) and nasal symptoms (change in TNSS: $-7.33 \pm 2.50$, paired $t$-test $\mathrm{p}<0.001$ ) of SAR patients. In 21 patients with coexisting asthma, the ACT score significantly increased from 16.10 to 22.57 on average (paired $t$-test $\mathrm{p}<0.001$ ), indicating better-controlled asthma. Using a threshold of $\geq 1$ point improvement in RQLQ overall score, $83.3 \%$ of patients responded to omalizumab. The responder group had a higher baseline RQLQ score and TNSS $(\mathrm{p}<0.05)$, but both responders and non-responders had comparable scores after treatment. Multiple linear regression analysis identified the baseline RQLQ overall score as a predictor of change in the RQLQ score in omalizumab-treated SAR.

Conclusion: Omalizumab is effective and safe in SAR treatment in a real-world setting. Keywords: omalizumab, seasonal allergic rhinitis, nasal symptoms score, quality of life, real world

\section{Introduction}

Allergic rhinitis (AR) is one of the most prevalent allergic diseases, characterized by the presence of inflammatory cells within mucosa and submucosa of the nasal cavity. ${ }^{1}$ The prevalence of AR ranges from $10 \% \sim 25 \%$ worldwide and $17 \% \sim 28.5 \%$ of adults in European populations. ${ }^{2}$ In China, a survey of self-reported AR over a 6-year period in the general Chinese adult population reported that the standardized prevalence of adult AR increased from $11.1 \%$ in 2005 to $17.6 \%$ in $2011 .^{3} \mathrm{We}$ have conducted an epidemiological study in the grassland of Northern China and found the prevalence of physician-diagnosed $\mathrm{AR}$ is $18.8 \%{ }^{4}$ The major clinical 
symptoms of AR include sneezing/and runny/stuffy/itchy nose, which are usually accompanied by red, itchy, and watery eyes. ${ }^{5,6}$ Because of its high prevalence and irritating symptoms, AR seriously affects life quality, work, or school attendance of patients. ${ }^{7,8}$ Furthermore, it has been shown that $\mathrm{AR}$ is associated with higher risk and poorer outcomes of asthma. ${ }^{9,10}$

AR is usually classified into seasonal (SAR), perennial (PAR) or persistent AR, intermittent (episodic) AR. ${ }^{11}$ SAR occurs in the pollen seasons when abundant airborne pollens exist in the environment. In this case, the sensitizing pollens contact with nasal mucosa and induce typical IgEmediated cascades of the inflammatory process.

Currently, antihistamines, corticosteroids, antileukotrienes, and immunotherapy, used alone or in combination, are the major clinical treatments for SAR. ${ }^{12-14}$ However, the effects of these drugs on the nasal and ocular symptoms of SAR vary greatly. Omalizumab is an anti-IgE monoclonal antibody that reduces the release of downstream inflammatory factors, blocking the inflammatory cascade after exposure to allergens. ${ }^{15}$ This therapeutic antibody has been officially approved for the treatment of moderate-to-severe allergic asthma, chronic spontaneous urticaria (CSU), severe chronic rhinosinusitis with nasal polyposis (CRSwNP), but not approved for SAR treatment by Federal Drug Administration (FDA) and European Medicines Agency (EMA). Randomized trials and real-life studies have shown that omalizumab can also significantly improve symptoms and QOL in patients with seasonal and perennial AR. ${ }^{16-19}$ A recent Phase III study demonstrated that omalizumab improved symptoms and quality of life in patients with severe cedar pollinosis, a common form of SAR in Japan. ${ }^{20}$ Based on this, omalizumab was approved for the treatment of severe cedar pollinosis in Japan in December 2019 (https:// www.novartis.co.jp/news/media-releases/prkk20191211).

However, there is still a lack of clinical data as to whether this therapeutic antibody can provide benefits to Chinese patients with SAR. We therefore investigated the clinical efficacy and response predictors of Chinese patients treated with omalizumab in a real-world setting.

\section{Patients and Methods}

\section{Patients and Study Design}

This is an observational study of omalizumab therapy in patients with SAR. Patients receiving anti-IgE (omalizumab) therapy were enrolled at Beijing Shijitan Hospital affiliated to Capital Medical University between June 2018 and March 2019. Approval to conduct this study was granted by the institutional review boards of Beijing Shijitan Hospital. Written informed consent was obtained from participants. For participants under the age of 18 years, a parent or legal guardian also gave an informed consent. This study was conducted in accordance with the Declaration of Helsinki.

The inclusion criteria were as follows: a) aged between 6 and 70 years old; b) diagnosed with SAR by physicians (conforming to the diagnostic criteria of AR; c) with $\geq 2$ years history of SAR, positive results of skin prick test, and/ or positive-specific IgE test for pollens) accompanied with or without asthma. Patients were excluded if they were allergic to the active substances of omalizumab, in pregnancy or lactation, or had heavily impaired lung and renal function, severe cardiovascular diseases, cancers, autoimmune diseases, or endocrine disorders, or had received antiIgE therapy before enrollment. Patients with simultaneous nasal spray drug, antihistamine, antileukotrienes, glucocorticoids, or allergen immunotherapy (AIT) were not excluded, and these therapies continued during the current study. A total of 16 kinds of pollens commonly seen in Northern China were tested by skin prick test (SPT) as previously described. $^{4}$

Total dosages of omalizumab were determined according to each patient's total serum IgE and body weight recommended by Xolair instrument Novartis. Omalizumab was administered subcutaneously every month.

\section{Treatment Parameters}

Data were prospectively collected at enrollment and posttreatment. The baseline demographic and clinical variables included age, sex, and body weight, duration of SAR, family history, allergic comorbidities, smoking, and total serum IgE. RQLQ and TNSS were used to assess the rhinitis response to omalizumab therapy, while ACT was applied to measure asthma response in patients with comorbid asthma.

The RQLQ is a self-administered questionnaire for the assessment of rhinitis-related quality of life (QOL) that contains 28 items in 7 domains (activities, sleep, non-nose /eye symptoms, practical problems, nasal problems, eye problems, and emotional function). ${ }^{21}$ Each item of the RQLQ is scored using a $0-6$ point scale, while the overall RQLQ score is the average of all items, and higher scores indicate much worse QOL. Patients were defined as responders if an improvement ( $\geq 1$ point) in overall RQLQ score was observed. ${ }^{22}$ The TNSS contains 4 items 
(rhinorrhea, nasal congestion, nasal itching, and sneezing), and each item is graded on a $0-3$ point scale, with a score of 3 being the most severe, possibly, resulting in a total score of 12 points.

The ACT (asthma control test) consists of five items: activity limitation, shortness of breath, awaking due to asthma symptoms, reliever medication, and global judgments of asthma control. Each item is scaled from 1 to 5 points and the total score of ACT is 25 .

\section{Statistical Analysis}

The significance of clinical changes from baseline to posttreatment was determined by paired $t$-test. Comparisons based on baseline characteristics between responders and non-responders were performed using the chi-square test for categorical variables and the $t$-test or Mann-Whitney $U$-test for continuous variables. Multiple linear regression analysis was performed to investigate the factors associated with the change in RQLQ. All analyses were conducted using SPSS 19.0 (IBM Corp, NY, USA). P values less than 0.05 indicated statistical significance.

\section{Results}

\section{Baseline Characteristics}

Patients with SAR ( $\mathrm{n}=60,25$ males and 35 females) who were administered anti-IgE therapy were enrolled (Table 1, Figure 1). The mean age was $35.47( \pm 17.02)$ years, with history duration of SAR varying from 2 to 40 years. Among these patients, 21 patients also had asthma, while 36 patients had a family history of SAR. The median dosage of omalizumab was 400 (range: 150 to 1800) mg in all patients, while the mean duration of omalizumab treatment was 2.9 months. The median initial omalizumab dose was $150 \mathrm{mg}$ (49 patients with $150 \mathrm{mg}, 8$ with $300 \mathrm{mg}, 2$ with $450 \mathrm{mg}$, 1 with $600 \mathrm{mg}$ ) per month. Patients were given omalizumab in the pollen season between June and September 2018 in our hospital in Beijing, when there were abundant pollens in the environment.

Other than omalizumab, patients had 0 to 5 additional medications for SAR treatment (mean=1.4). The percentages of patients having AIT, oral antihistamines, antileukotrienes, and intranasal glucocorticoids were 20 (32.8\%), $33(55.0 \%), 17$ (28.3\%), and 12 (20.0\%), respectively. The percentage of patients with comorbid asthma who also used corticosteroid/long-acting beta-agonist (ICS/LABA) for asthma control was $61.9 \%$.
Table I Basic Characteristic of SAR Patients

\begin{tabular}{|c|c|}
\hline Variables & $N=60$ \\
\hline Age, years & $35.47( \pm 17.02)$ \\
\hline Sex (male/female), $\mathrm{n}$ & $25 / 35$ \\
\hline BMI, $\mathrm{kg} / \mathrm{m}^{2}$ & $23.16( \pm 4.20)$ \\
\hline Body weight, kg & $62.3( \pm 16.1)$ \\
\hline \multicolumn{2}{|l|}{ Comorbidity, n (\%) } \\
\hline Asthma & $21(35 \%)$ \\
\hline Food allergy & $3(5 \%)$ \\
\hline Eczema & $2(3.3 \%)$ \\
\hline Family history, n (\%) & $36(60 \%)$ \\
\hline Pollen season, $n(\%)$ & $34(56.7 \%)$ \\
\hline Smoking, n (\%) & $4(6.7 \%)$ \\
\hline Intermittent/Persistent rhinitis, $\mathrm{n}$ & $31 / 29$ \\
\hline Disease duration, years & $10(1,40)$ \\
\hline Serum IgE, IU/mL & $213(|6.8,2| 4 \mid)$ \\
\hline Total dosage of omalizumab, $\mathrm{mg}$ & $450(150,1800)$ \\
\hline Duration of treatment, months & $2.9( \pm 1.2)$ \\
\hline Baseline RQLQ overall score & $2.55( \pm 1.04)$ \\
\hline Activities & $3.42( \pm 1.55)$ \\
\hline Sleep & $2.72( \pm 1.62)$ \\
\hline Non-nose/eye symptoms & $2.30( \pm 1.46)$ \\
\hline Practical problems & $3.62( \pm 1.37)$ \\
\hline Nasal problems & $3.84( \pm 1.36)$ \\
\hline Eye problems & $1.42( \pm 1.09)$ \\
\hline Emotional function & $1.25( \pm 1.24)$ \\
\hline Baseline TNSS & $9.97( \pm 2.29)$ \\
\hline Number of respiratory medications & $1.4( \pm 1.2)$ \\
\hline AIT & $20(32.8 \%)$ \\
\hline Antihistamines (i.n.) & $\mathrm{I}(\mathrm{I} .7 \%)$ \\
\hline Antihistamines (p.o.) & $33(55.0 \%)$ \\
\hline Antileukotrienes (p.o.) & $17(28.3 \%)$ \\
\hline Glucocorticoids (i.n.) & $12(20.0 \%)$ \\
\hline \multicolumn{2}{|l|}{ Asthma patients $(\mathrm{N}=2 \mathrm{I})$} \\
\hline Baseline ACT score & $16.10( \pm 4.12)$ \\
\hline ICS/LABA & $13(6 \mid .9 \%)$ \\
\hline
\end{tabular}

Note: Continuous variables are presented as mean ( \pm standard deviation) or median (range).

Abbreviations: SAR, seasonal allergic rhinitis; BMI, body mass index; RQLQ, Rhinoconjunctivitis Quality of Life Questionnaire; TNSS, Total Nasal Symptoms Score; ACT, Asthma Control Test; AIT, allergen immunotherapy; i.n., intranasal drugs; p.o., oral drugs; ICS/LABA, inhaled corticosteroid/long-acting beta-agonist.

As summarized in Supplementary Table 1, the most common allergic pollen was Artemisia sieversiana that was positive in $86.7 \%(52 / 60)$ of patients by SPT. The positivity rate of the other 15 pollens ranged from $16.7 \%$ to $40.0 \%$.

\section{Quality of Life and Symptom Scores}

The QOL and symptoms of SAR patients improved significantly after omalizumab treatment (Table 2). The 


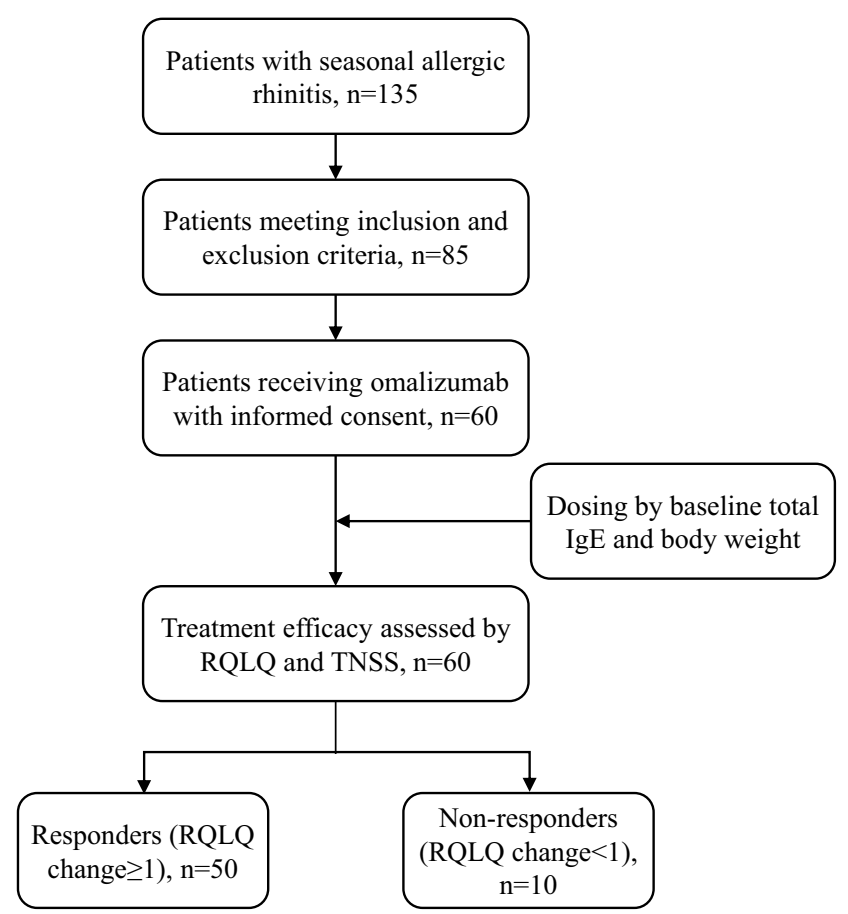

Figure I Flow-chart of study design.

Abbreviations: RQLQ, Rhinoconjunctivitis Quality of Life Questionnaire; TNSS, Total Nasal Symptoms Score.

RQLQ overall score changed from $-2.55( \pm 1.04)$ to 0.47 $( \pm 0.47)$, with a substantial reduction of $2.08( \pm 1.01)$ points (paired $t$-test, $\mathrm{p}<0.001$ ). The scores of each domain of RQLQ also showed significant reduction $(\mathrm{p}<0.001)$. In addition, nasal symptoms were obviously relieved after omalizumab treatment, as indicated by the reduction of TNSS $(7.33 \pm 2.50, \mathrm{p}<0.001)$. For SAR patients with asthma, an increase of ACT score from 16.10 to 22.57 was observed after anti-IgE treatment, and 19/21 (90.5\%) patients were well-controlled for asthma (ACT score $\geq 20$ ).

\section{Response Analysis}

Using an improvement threshold of $\geq 1$ point in the RQLQ overall score, $50(83.3 \%)$ patients with SAR responded to omalizumab while 10 subjects with SAR did not. There were no differences between responders and nonresponders in baseline characteristics (Table 3). However, the responders had higher baseline RQLQ overall score $(2.83 \pm 0.90$ versus $1.15 \pm 0.37, \mathrm{p}<0.001)$ and higher TNSS $(10.28 \pm 2.11$ versus $8.40 \pm 2.63, \mathrm{p}=0.017)$ than nonresponders. After treatment, no further significant differences in RQLQ overall score or TNSS were observed between these two groups ( $\mathrm{p}=0.951$ and 0.566 , respectively). Univariate logistic regression showed that patients with higher baseline RQLQ overall score were more likely to respond to omalizumab (OR=117, 95\% CI: 4.3-3193).

\section{Response Predictors}

Multiple linear regression analysis of baseline characteristics was performed to identify factors that had significant impact on RQLQ change during omalizumab treatment (Table 4). Baseline RQLQ overall score was an independent predictor of the RQLQ response to omalizumab $(p<0.001)$. In contrast, other factors including age, sex, comorbid asthma, family history, treatment in the pollen season, total dosage of omalizumab, total serum IgE, and AIT were not associated with RQLQ changes ( $p>0.1)$.

\section{Discussion}

IgE plays a pivotal role in the development of allergic rhinitis, as well as atopic asthma, urticaria, atopic dermatitis, and food allergy. ${ }^{23}$ Omalizumab is a humanized monoclonal antibody that specifically binds to $\operatorname{IgE}$, reduces serum-free IgE, and down-regulates the high-

Table 2 Changes in RQLQ, TNSS, and ACT Scores from Pre- to Post-Treatment of Omalizumab

\begin{tabular}{|c|c|c|c|c|}
\hline Scores & Pre-Treatment & Post-Treatment & Change & $\mathbf{p}^{\#}$ \\
\hline RQLQ overall score & $2.55( \pm 1.04)$ & $0.47( \pm 0.47)$ & $-2.08( \pm 1.01)$ & $<0.001$ \\
\hline Activities & $3.42( \pm 1.55)$ & $0.76( \pm 0.73)$ & $-2.66( \pm 1.54)$ & $<0.001$ \\
\hline Sleep & $2.72( \pm 1.62)$ & $0.28( \pm 0.56)$ & $-2.44(1.64)$ & $<0.001$ \\
\hline Non-nose/eye symptoms & $2.30( \pm 1.46)$ & $0.40( \pm 0.53)$ & $-1.89( \pm 1.45)$ & $<0.001$ \\
\hline Practical problems & $3.62( \pm 1.37)$ & $0.69( \pm 0.77)$ & $-2.92( \pm I .4 I)$ & $<0.001$ \\
\hline Nasal problems & $3.84( \pm 1.36)$ & $0.87( \pm 0.98)$ & $-2.97( \pm 1.27)$ & $<0.001$ \\
\hline Eye problems & $1.42( \pm 1.09)$ & $0.28( \pm 0.46)$ & $-1.13( \pm 1.08)$ & $<0.001$ \\
\hline Emotional function & $1.25( \pm 1.24)$ & $0.15( \pm 0.39)$ & $-1.10( \pm 1.20)$ & $<0.001$ \\
\hline TNSS & $9.97( \pm 2.29)$ & $2.63( \pm 2.13)$ & $-7.33( \pm 2.50)$ & $<0.001$ \\
\hline ACT score ${ }^{\&}$ & $16.10( \pm 4.12)$ & $22.57( \pm 2.29)$ & $6.48( \pm 4.17)$ & $<0.001$ \\
\hline
\end{tabular}

Notes: ${ }^{\#}$ paired $t$-test; ${ }^{\&}$ only for 21 patients with asthma.

Abbreviations: RQLQ, Rhinoconjunctivitis Quality of Life Questionnaire; TNSS, Total Nasal Symptoms Score; ACT, Asthma Control Test. 
Table 3 Comparison of Baseline Characteristics and Treatment Efficacy Between Responders and Non-Responders to Omalizumab

\begin{tabular}{|c|c|c|c|}
\hline Variables & Responders $(\mathrm{N}=50)$ & Non-Responders $(\mathrm{N}=10)$ & $\mathbf{p}$ \\
\hline Age, years & $35.54( \pm \mid 7.21)$ & $35.10( \pm 16.91)$ & 0.941 \\
\hline Sex (male/female), $n$ & $19 / 3 \mid$ & $6 / 4$ & 0.198 \\
\hline BMI, $\mathrm{kg} / \mathrm{m}^{2}$ & $23.29( \pm 4.42)$ & $22.46( \pm 2.88)$ & 0.573 \\
\hline Body weight, kg & $62.66( \pm 16.59)$ & $60.30( \pm 13.84)$ & 0.676 \\
\hline Asthma $(\mathrm{Y} / \mathrm{N})$ & $18 / 32$ & $3 / 7$ & 0.717 \\
\hline Family history $(\mathrm{Y} / \mathrm{N})$ & $31 / 19$ & $5 / 5$ & 0.480 \\
\hline Pollen season $(\mathrm{Y} / \mathrm{N})$ & $27 / 23$ & $7 / 3$ & 0.351 \\
\hline Intermittent/Persistent rhinitis & $27 / 23$ & $4 / 6$ & 0.419 \\
\hline Disease duration, years & $10(5,10)$ & $8(5,13.8)$ & 0.902 \\
\hline Serum $\lg E, I U / m L$ & $220(138,454)$ & $132(103,226)$ & 0.154 \\
\hline Total dosage of omalizumab, $\mathrm{mg}$ & $450(300,600)$ & $450(338,450)$ & 0.783 \\
\hline Number of medications & $\mathrm{I} .4( \pm \mathrm{I} .2)$ & $\mathrm{I} .4( \pm \mathrm{I} .3)$ & 0.962 \\
\hline AIT $(\mathrm{Y} / \mathrm{N})$ & $16 / 34$ & $4 / 6$ & 0.624 \\
\hline \multicolumn{4}{|l|}{ RQLQ overall scores } \\
\hline Baseline & $2.83( \pm 0.90)$ & $1.15( \pm 0.37)$ & $<0.001$ \\
\hline Post-treatment & $0.47( \pm 0.48)$ & $0.48( \pm 0.40)$ & 0.951 \\
\hline \multicolumn{4}{|l|}{ TNSS } \\
\hline Baseline & $10.28( \pm 2.11)$ & $8.40( \pm 2.63)$ & 0.017 \\
\hline Post-treatment & $2.56( \pm 2.03)$ & $3.00( \pm 2.67)$ & 0.556 \\
\hline
\end{tabular}

Note: Chi-square test, $t$-test, or Mann-Whitney $U$-test were used as appropriate.

Abbreviations: BMI, body mass index; RQLQ, Rhinoconjunctivitis Quality of Life Questionnaire; TNSS, Total Nasal Symptoms Score; AIT, allergen immunotherapy.

Table 4 Multiple Linear Regression Analysis of Baseline Characteristics to Predict RQLQ Response to Omalizumab

\begin{tabular}{|l|l|l|l|l|}
\hline Variables & Coefficient & S.E. & t & p \\
\hline Age, years & 0.007 & 0.005 & 1.514 & 0.137 \\
Sex (male) & 0.182 & 0.156 & 1.165 & 0.250 \\
Comorbid asthma & -0.043 & 0.144 & -0.299 & 0.766 \\
Body weight, kg & -0.010 & 0.006 & -1.667 & 0.102 \\
Family history & -0.133 & 0.140 & -0.954 & 0.345 \\
Total dosage of omalizumab ( $\geq 600 \mathrm{mg})$ & -0.162 & 0.162 & -1.001 & 0.322 \\
Total serum IgE (IU/mL) & 0.000 & 0.000 & 0.317 & 0.753 \\
AIT & 0.200 & 0.139 & 1.442 & 0.156 \\
Baseline RQLQ overall score & -0.807 & 0.069 & -11.631 & $<0.001$ \\
\hline
\end{tabular}

Abbreviations: RQLQ, Rhinoconjunctivitis Quality of Life Questionnaire; AIT, allergen immunotherapy.

affinity receptor of the Fc portion of $\operatorname{IgE}(\mathrm{Fcc} R \mathrm{I})$ on the surface of immune cells. ${ }^{24,25}$ This antibody can block the degranulation of mast cells and basophils and consequent release of inflammatory factors by other immune cells and structural cells in mucosal tissues, which has made it a focus for clinical therapy of allergic diseases. The treatment efficacy of omalizumab in asthma patients has been determined to reduce exacerbations, rescue the usage of glucocorticoids and short-acting $\beta 2$ receptor agonist, and improve lung function, asthma control, and quality of life. $^{26-28}$ Nearly half of patients with severe atopic dermatitis had good response to omalizumab with $>50 \%$ reduction of severity scoring for their condition. ${ }^{29}$

Similarly, randomized clinical trials have shown that AR patients may also benefit greatly from omalizumab. ${ }^{30}$ This therapeutic antibody could significantly relieve the nasal symptoms and improve QOL, reducing usage of antihistamine medications in a dose-dependent manner in patients with moderate to severe ragweed-induced SAR. ${ }^{16}$ Omalizumab may improve nasal as well as ocular symptoms in patients with cedar pollen-induced SAR. Although injection site reactions have been observed, ${ }^{18}$ the 
incidence of adverse events in omalizumab group is comparable to the control group, ${ }^{28}$ indicating that omalizumab might be safe for AR patients.

Through prospective observation in a real-life setting, we found that SAR patients obtained benefits from omalizumab treatment in clinical practice. Patients received monthly doses of omalizumab based on individual body weight and total serum $\operatorname{IgE}$ at recruitment referenced to previous trials. ${ }^{31-33}$ After 1 to $6($ mean $=2.9)$ months of treatment, SAR patients had a statistically significant mean reduction of 2.08 points in their RQLQ overall score, and mean improvements of $>1.00$ in all domains. Omalizumab also significantly relieved nasal symptoms, supported by a mean reduction of 7.33 in TNSS scores. Taken together, the data suggest that treatment with omalizumab has considerable efficacy on Chinese patients with SAR.

AR and asthma are usually comorbid with each other. Epidemiology investigations show that $60 \% \sim 80 \%$ of asthma patients have rhinitis, while $20 \% \sim 40 \%$ of rhinitis patients also have asthma. ${ }^{34}$ Since allergic rhinitis and allergic asthma share a similar pathogenesis, patients with either condition may gain similar clinical benefits from treatment with omalizumab. A previous study has shown that an asthma response to omalizumab predicts a better rhinitis response to the same treatment. ${ }^{22}$ Other studies suggest that co-existing rhinitis might affect the clinical outcomes of asthma patients. ${ }^{35}$ In the present study, 21 SAR patients had asthma and also received omalizumab treatment. Co-existing asthma of SAR patients did not affect the quality of life (RQLQ overall score, $\mathrm{p}=0.895$ ) or symptoms (TNSS, $\mathrm{p}=0.880$ ) at baseline. After treatment, asthma patients had significant improvement of ACT scores. However, between SAR patients with or without asthma, omalizumab treatment showed a similar effect on the reduction of RQLQ overall score (LS mean difference: $0.103,95 \%$ CI: -0.449 to 0.654 , $\mathrm{p}=0.711$ ) and TNSS (LS mean difference: $0.220,95 \%$ CI: -1.582 to $1.142, p=0.748)$, indicating that co-existing asthma did not influence the clinical outcomes of SAR.

It is clearly important to identify predictors of response to omalizumab for better management of SAR in clinical practice. In the present study, $83.3 \%(50 / 60)$ of patients treated with omalizumab had clinically significant improvement ( $\geq 1.00$-point reduction in RQLQ overall score). Furthermore, the responders group had higher baseline RQLQ overall score and TNSS than the nonresponders group. However, no further differences in these scores were observed after omalizumab treatment between both groups, and mean scores were all quite low $(<1.00$ point in RQLQ, $\leq 3.00$ points in TNSS). These results suggest that almost all patients treated with omalizumab can achieve high quality of life and remission of symptoms, regardless of their baseline scores, though they may not fulfill the criteria of response. Multiple linear regression analysis revealed that baseline RQLQ overall score was associated with reduction of RQLQ score $(p<0.001)$, suggesting that patients who had poor quality of life (ie, higher RQLQ score) at baseline might benefit more from omalizumab. This is similar to a previous study which identified baseline ACQ-5 to predict magnitude of change in ACQ-5 in omalizumab-treated allergic asthma. ${ }^{36}$ Besides baseline scores, we did not observe any additional predictors for treatment efficacy of omalizumab.

Previous studies suggested that allergic rhinitis is tightly associated with type 2 inflammation response and that IL-4, IL-5 as well as IL-13 play important roles in AR pathogenesis. ${ }^{37}$ Type 2 inflammation is characterized by the overproduction of type 2 cytokines (IL-4, IL-5, IL-13) released by Th2 cells, which may stimulate Type $2 \mathrm{immu}-$ nity featured by elevated $\operatorname{IgE}$ level and eosinophils infiltrating. ${ }^{38}$ Type 2 inflammation pathway plays critical roles in pathogenesis of many allergic diseases, including asthma, chronic rhinosinusitis, chronic idiopathic urticaria. $^{39,40}$ Biologics targeting this pathway (anti-IgE, anti-IL4, anti-IL5/13) show predominant treatment effect and some have been approved for clinical use. ${ }^{41}$ Some type 2 biomarkers are developed to monitor response to these biologics. Omalizumab is an anti-IgE monoclonal antibody licensed for use in severe allergic asthma and CRSwNP. The post hoc analysis of a randomized study for severe asthma patients treated by omalizumab showed that blood eosinophils, serum periostin, and fractional exhaled nitric oxide (FeNO) predicted the risk for exacerbations after treatment. ${ }^{42}$ This indicates a predictive role of these biomarkers for response to omalizumab. However, whether these markers are associated with omalizumab efficacy in treating allergic rhinitis remains unclear. Our study showed baseline IgE level was not a predictor for response to omalizumab. But we did not test blood eosinophils, serum periostin, and fractional exhaled nitric oxide (FeNO), thus failed to explore the predictive role of these type 2 biomarkers in allergic rhinitis patients treated with omalizumab.

Notably, we only detected the seasonal allergen but whether there is co-existing perennial allergen was unclear. In addition, most of the patients were tested positive for 
several seasonal allergen. Therefore, the efficacy of omalizumab in patients sensitized to only seasonal allergen or to a certain pollen allergen was unable to be analyzed. The influence of the pattern of sensitization profile on omalizumab efficacy in AR needs further investigation.

In conclusion, our data show the effectiveness of omalizumab for treatment of Chinese patients with seasonal allergic rhinitis in real-world clinical practice. Almost all patients achieved good clinical outcomes regardless of their baseline characteristics, including comorbid asthma, baseline scores, simultaneous therapies, and omalizumab dosage.

\section{Abbreviations}

SAR, seasonal allergic rhinitis; QOL, quality of life; RQLQ, Rhinoconjunctivitis Quality of Life Questionnaire; TNSS, Total Nasal Symptoms Score; ACT, Asthma Control Test.

\section{Author Contributions}

All authors made substantial contributions to conception and design, acquisition of data, analysis and interpretation of data; took part in drafting the article and revising it critically for important intellectual content; agreed to submit to the current journal; gave final approval of the version to be published; and agree to be accountable for all aspects of the work.

\section{Funding}

This work was supported by the National Key Specialty Funding of China granted to XW, Beijing Municipal Administration of Hospitals Clinical Medicine Development of Special Funding Support (No. ZYLX201826), Open Research Funding of Beijing Key Laboratory of Biocharacteristic Profiling for Evaluation of Rational Drug Use (No. 2019-KF11) and Science and Technology Research and Development Project of China National Railway Group Co. (No. J2019Z603) granted to TM.

\section{Disclosure}

All authors declare no conflicts of interest.

\section{References}

1. Khan David A. Allergic rhinitis and asthma: epidemiology and common pathophysiology. Allergy Asthma Proc. 2014;35(5):357-361. doi:10.2500/aap.2014.35.3794

2. Testa D, DI Bari M, Nunziata M, et al. Allergic rhinitis and asthma assessment of risk factors in pediatric patients: A systematic review. Int J Pediatr Otorhinolaryngol. 2020;129:109759. doi:10.1016/j. ijporl.2019.109759
3. Wang XD, Zheng M, Lou HF, et al. An increased prevalence of self-reported allergic rhinitis in major Chinese cities from 2005 to 2011. Allergy. 2016;71(8):1170-1180. doi:10.1111/all.12874

4. Wang X-Y, Ma -T-T, Wang X-Y, et al. Prevalence of pollen-induced allergic rhinitis with high pollen exposure in grasslands of Northern China. Allergy. 2018;73(6):1232-1243. doi:10.1111/all.13388

5. Kulig M, Klettke U, Wahn V, Forster J, Bauer C-P, Wahn U. Development of seasonal allergic rhinitis during the first 7 years of life. J Allergy Clin Immunol. 2000;106(5):832-839. doi:10.1067/ mai.2000.110098

6. Pastacaldi C, Lewis P, Howarth P. Staphylococci and staphylococcal superantigens in asthma and rhinitis: a systematic review and meta-analysis. Allergy. 2011;66(4):549-555. doi:10.1111/j.13989995.2010.02502.x

7. Valero A, et al. Development and validation of a new Spanish instrument to measure health-related quality of life in patients with allergic rhinitis: the ESPRINT Questionnaire. Value Health. 2007;10 (6):466-477. doi:10.1111/j.1524-4733.2007.00202.x

8. Colás C, Brosa M, Antón E, et al. Estimate of the total costs of allergic rhinitis in specialized care based on real-world data: the FERIN Study. Allergy. 2017;72(6):959-966. doi:10.1111/all.13099

9. Johnston NW. Asthma exacerbations \{middle dot\} 1: epidemiology. Thorax. 2006;61(8):722-728. doi:10.1136/thx.2005.045161

10. Bousquet J, Schünemann HJ, Samolinski B, et al. Allergic rhinitis and its impact on asthma (ARIA): achievements in 10 years and future needs. J Allergy Clin Immunol. 2012;130(5):1049-1062. doi:10.1016/j.jaci.2012.07.053

11. Wallace DV, Dykewicz MS, Bernstein DI, et al. The diagnosis and management of rhinitis: an updated practice parameter. J Allergy Clin Immunol. 2008;122(2):S1-S84. doi:10.1016/j.jaci.2008.06.003

12. Juel-Berg N, Darling P, Bolvig J, et al. Intranasal corticosteroids compared with oral antihistamines in allergic rhinitis: A systematic review and meta-analysis. Am J Rhinol Allergy. 2017;31(1):19-28. doi:10.2500/ajra.2016.30.4397

13. Meltzer EO. Pharmacotherapeutic strategies for allergic rhinitis: matching treatment to symptoms, disease progression, and associated conditions. Allergy Asthma Proc. 2013;34(4):301-311. doi:10.2500/ aap.2013.34.3676

14. Wei C. The efficacy and safety of H1-antihistamine versus Montelukast for allergic rhinitis: A systematic review and meta-analysis. Biomed Pharmacother. 2016;83:989-997. doi:10.1016/j.biopha.2016.08.003

15. Nyborg AC, Zacco A, Ettinger R, et al. Development of an antibody that neutralizes soluble $\operatorname{IgE}$ and eliminates $\mathrm{IgE}$ expressing B cells. Cell Mol Immunol. 2016;13(3):391-400. doi:10.1038/cmi.2015.19

16. Casale TB, Condemi J, Laforce C, et al. Effect of omalizumab on symptoms of seasonal allergic rhinitis: a randomized controlled trial. JAMA. 2001;19:2956-2967. doi:10.1001/jama.286.23.2956

17. Kuehr J, Brauburger J, Zielen S, et al. Efficacy of combination treatment with anti-IgE plus specific immunotherapy in polysensitized children and adolescents with seasonal allergic rhinitis. J Allergy Clin Immunol. 2002;109(2):274-280. doi:10.1067/mai.2002.121949

18. Okubo K, Ogino S, Nagakura T, Ishikawa T. Omalizumab is effective and safe in the treatment of Japanese cedar pollen-induced seasonal allergic rhinitis. Allergol Int. 2006;55(4):379-386. doi:10.2332/ allergolint.55.379

19. Cavaliere C, Begvarfaj E, Incorvaia C, et al. Long-term omalizumab efficacy in allergic rhinitis. Immunol Lett. 2020;227:81-87. doi:10.1016/j.imlet.2020.08.002

20. Okubo K, Okano M, Sato N, et al. Add-on omalizumab for inadequately controlled severe pollinosis despite standard-of-care: a randomized study. J Allergy Clin Immunol Pract. 2020;8 (3130):3130-3140.e2. doi:10.1016/j.jaip.2020.04.068

21. Juniper EF, Guyatt GH. Development and testing of a new measure of health status for clinical trials in rhinoconjunctivitis. Clin Exp Allergy. 1991;21(1):77-83. doi:10.1111/j.1365-2222.1991.tb00807.x 
22. Humbert M, Boulet LP, Niven RM, Panahloo Z, Blogg M, Ayre G. Omalizumab therapy: patients who achieve greatest benefit for their asthma experience greatest benefit for rhinitis. Allergy. 2009;64 (1):81-84. doi:10.1111/j.1398-9995.2008.01846.x

23. Kowalski ML, Ansotegui I, Aberer W, et al. Risk and safety requirements for diagnostic and therapeutic procedures in allergology: world Allergy Organization Statement. World Allergy Organ J. 2016;9:33. doi:10.1186/s40413-016-0122-3

24. Licari A, Marseglia G, Castagnoli R, Marseglia A, Ciprandi G. The discovery and development of omalizumab for the treatment of asthma. Expert Opin Drug Discov. 2015;10(9):1033-1042. doi:10.1517/17460441.2015.1048220

25. Bousquet J, Cabrera P, Berkman N, et al. The effect of treatment with omalizumab, an anti-IgE antibody, on asthma exacerbations and emergency medical visits in patients with severe persistent asthma. Allergy. 2005;60(3):302-308. doi:10.1111/j.1398-9995.2004.00770.x

26. Busse WW, Morgan WJ, Gergen PJ, et al. Randomized trial of omalizumab (anti-IgE) for asthma in inner-city children. $N$ Engl $J$ Med. 2011;364(11):1005-1015. doi:10.1056/NEJMoa1009705

27. Li J, Kang J, Wang C, et al. Omalizumab improves quality of life and asthma control in Chinese patients with moderate to severe asthma: A randomized phase III study. Allergy Asthma Immunol Res. 2016;8 (4):319-328. doi:10.4168/aair.2016.8.4.319

28. Wang HH, Li YC, Huang YC. Efficacy of omalizumab in patients with atopic dermatitis: A systematic review and meta-analysis. $J$ Allergy Clin Immunol. 2016;138:1719-1722. doi:10.1016/j. jaci.2016.05.038

29. Tsabouri S, Tseretopoulou X, Priftis K, Ntzani EE. Omalizumab for the treatment of inadequately controlled allergic rhinitis: a systematic review and meta-analysis of randomized clinical trials. J Allergy Clin Immonol Pract. 2014;2:332-340. doi:10.1016/j.jaip.2014.02.001

30. Kamin W, Kopp MV, Erdnuess F, Schauer U, Zielen S, Wahn U. Safety of anti-IgE treatment with omalizumab in children with seasonal allergic rhinitis undergoing specific immunotherapy simultaneously. Pediatr Allergy Immunol. 2010;21(1-Part-II):e160e165. doi:10.1111/j.1399-3038.2009.00900.x

31. Klunker S, Saggar LR, Seyfert-Margolis V, et al. Combination treatment with omalizumab and rush immunotherapy for ragweed-induced allergic rhinitis: inhibition of $\mathrm{IgE-facilitated} \mathrm{aller-}$ gen binding. $J$ Allergy Clin Immunol. 2007;120(3):688-695. doi:10.1016/j.jaci.2007.05.034
32. Kopp MV, Hamelmann E, Zielen S, et al. Combination of omalizumab and specific immunotherapy is superior to immunotherapy in patients with seasonal allergic rhinoconjunctivitis and co-morbid seasonal allergic asthma. Clin Exp Allergy. 2009;39(2):271-279. doi:10.1111/j.1365-2222.2008.03121.x

33. Togias A. Rhinitis and asthma: evidence for respiratory system integration. $J$ Allergy Clin Immunol. 2003;111(6):1171-1183. doi:10.1067/mai.2003.1592

34. Price D, Zhang Q, Kocevar VS, Yin DD, Thomas M. Effect of a concomitant diagnosis of allergic rhinitis on asthma-related health care use by adults. Clin Exp Allergy. 2005;35(3):282-287. doi:10.1111/j.1365-2222.2005.02182.x

35. Bousquet J, Gaugris S, Kocevar VS, et al. Increased risk of asthma attacks and emergency visits among asthma patients with allergic rhinitis: a subgroup analysis of the improving asthma control trial. Clin Exp Allergy. 2005;35(6):723-727. doi:10.1111/j.1365-2222.2005.02251.x

36. Gibson PG, Reddel H, McDonald VM, et al. Effectiveness and response predictors of omalizumab in a severe allergic asthma population with a high prevalence of comorbidities: the Australian Xolair Registry. Int Med J. 2016;46(9):1054-1062. doi:10.1111/imj.13166

37. Shi Z, Xu M, Chen X, Wang J, Zhao T, Zha D. The regulatory role of SFRP5/WNT5A axis in allergic rhinitis through inhibiting JNK pathway activation and lowering mucin generation in human nasal epithelial cells.. Exp Mol Pathol. 2020;118:104591. doi:10.1016/j. yexmp.2020.104591

38. Fahy J. Type 2 inflammation in asthma - present in most, absent in many. Nat Rev Immunol. 2015;15(1):57-65. doi:10.1038/nri3786

39. Dunican E, Fahy J. The Role of Type 2 Inflammation in the Pathogenesis of Asthma Exacerbations.. Ann Am Thorac Soc. 2015;12 Suppl 2(Suppl 2):S144. doi:10.1513/AnnalsATS.201506-377AW

40. Riccio A, Tosca M, Cosentino C, et al. Cytokine pattern in allergic and non-allergic chronic rhinosinusitis in asthmatic children. Clin Exp Allergy. 2002;32(3):422-426. doi:10.1046/j.1365-2222.2002.01315.x

41. Manka L, Wechsler M. New biologics for allergic diseases. Expert Rev Clin Immunol. 2018;14(4):285-296. doi:10.1080/1744666X.20 18.1459188

42. Hanania N, Wenzel S, Rosen K, et al. Exploring the effects of omalizumab in allergic asthma: an analysis of biomarkers in the EXTRA study. Am J Respir Crit Care Med. 2013;187(8):804-811. doi:10.1164/rccm.201208-1414OC

\section{Publish your work in this journal}

The Journal of Asthma and Allergy is an international, peer-reviewed open-access journal publishing original research, reports, editorials and commentaries on the following topics: Asthma; Pulmonary physiology; Asthma related clinical health; Clinical immunology and the immunological basis of disease; Pharmacological interventions and new therapies. The manuscript management system is completely online and includes a very quick and fair peer-review system, which is all easy to use. Visit http://www.dovepress.com/testimonials.php to read real quotes from published authors. 